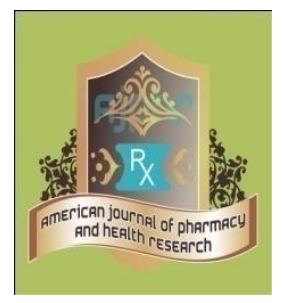

\title{
Application Of Solid Phase Micro-Extraction For The Analysis Of Organophosphorus Pesticide Residue: A Review
}

\author{
Tania Debnath, Anees Ahmad Siddiqui* \\ Department of Pharmaceutical Chemistry, School of Pharmaceutical Education and Research, \\ Jamia Hamdard, New Delhi-110062
}

\begin{abstract}
In the review, the focus has been laid on discussing various modes of solid phase microextraction for sample pre treatment and sample pre concentration of organophosphorus pesticide residue from the complex matrix they are embedded in. Wider availability of organophosphorus pesticide residue in environment pose a threat of toxicity, so quantification of the same becomes important. Sample preparation is one of the initial steps in any analytical procedure, which is quite exhaustive and hence leads to increase in error. So, over the years, the focus has been to minimize the burden on this step, thereby introducing many modifications in existing sample preparation techniques available, which are serially discussed. One such modification is miniaturization and automation of solid phase extraction, various developments of which so far has been discussed especially considering techniques employed for OPP residue extraction. Starting from stir bar sorptive extraction to newer techniques such as use of sol gel sorbents. Their deployment in sample pre concentration for extraction of organophosphorus pesticide residue; a comparison among the modes used, challenges and future trend in modification of solid phase micro extraction with emphasis on incorporating the usefulness to switch over to green analytical chemistry is discussed. Keywords: Organophosphorus pesticide (OPP), Solid phase extraction (SPE), Stir Bar Sorptive Extraction (SBSE), Dispersive solid phase extraction (dSPE), Dispersive solid phase microextraction (d- $\mu \mathrm{SPE})$, Microextraction by packed sorbent (MEPS), Fabric phase sorptive extraction (FPSE).
\end{abstract}

*Corresponding Author Email: aasiddiqui@jamiahamdard.ac.in

Received 10 June 2020, Accepted 06 July 2020

Please cite this article as: Siddiqui AA et al., Application Of Solid Phase Micro-Extraction For The Analysis Of Organophosphorus Pesticide Residue: A Review. American Journal of Pharmacy \& Health Research 2020. 


\section{INTRODUCTION}

Sample preparation refers to the ways by which a sample is treated before its analyses. It is a vital part of any analytical procedure. This is the foremost crucial and time taking method and needs skill to execute action so as to minimize the probabilities of error ${ }^{1}$. It is additionally necessary to maintain excellent care to confirm the proper isolation of the interest analytes within a complex matrix, to develop reliable ways and consequently get higher sensitivity, precision, accuracy, and recovery within the results ${ }^{2}$.

The well-known traditional extraction techniques utilized in analytical chemistry involves liquidliquid extraction (LLE) and solid phase extraction (SPE). However, in the recent event of progression miniaturized sample preparation approaches has been growing considerably in recent years. In general, these miniaturized techniques are modifications of those conventional ones, mentioned previously. Along with this compaction of sample preparation methods, a focus on green analytical chemistry is also widening to be considered vital in such various technique modifications ${ }^{3-4}$. The approach lies in the fact of minimising the use of hazardeous solvents in the pre-treatments.

Furthermore, many different advantages, like a lot of easy procedure, faster analysis, higher extraction performance, and reduced quantity of sample needed, can be regarded as potential blessings of miniaturization and automation. Another significant contribution to the development and spread of the miniaturized techniques, involve the current advances in both the use of greener extraction solvents accompanied with the synthesis of new sorbent materials. Both approaches represent alternative methodology which aims to acquire better analytical results ${ }^{5-6}$. Sample preparation is one of the important steps in pesticide residue analysis. But due to complex matrix, the extraction conditions become cumbersome which often presents the risk of inaccuracies causing hinderance in accurate analysis. Miniaturisation and automation of sample preparation has been proved to be boon for such critical analyses, along with keeping a check on environment by following the view of green chemistry ${ }^{7}$.

In the present review, an attempt is made to study the miniaturised sample preparation techniques employed so far for isolating organophosphorus(OPP) pesticide residues from their complex matrix, especially focusing on the different modes of solid phase Microextraction (SPME), including discussion on stir bar sorptive extraction(SBSE), dispersive solid phase extraction(dSPE), dispersive solid phase microextraction(d- $\mu \mathrm{SPE})$, ME by packed sorbent (MEPS) along with a brief discussion on newer variation i.e. fabric phase sorptive extraction based 
upon sol gel sorbent. Also, a comparison on widely used technique and relevant reason supporting its utility for the same.

\section{Solid Phase Microextraction}

SPME is a commonly used sorbent based microextraction technique in today's world which imparts analyte partition and establishes a state of equilibrium between the analyte in the sample and the stationary phase of the coated fused silica, which can either be solid or liquid particles suspended in liquid polymer or can be a combination of both ${ }^{8}$. The credit for its creation goes to the researchers Pawliszyn and Arthur who in the early nineties utilized the SPME technique to extract organic micro-pollutants from environmental samples ${ }^{9}$. This miniaturised method has find its usage in various sector involving analysis in food, agriculture, environmental pollutants, radioactivity. Over the years, researches focussing on developing more selective and specific fibers has been the centre of interest in most studies in this area. Better specificity for target analytes and relevancy for samples with complex matrix compositions are the significant challenges to be overcome in SPME ${ }^{10}$.

\section{Stir Bar Sorptive Extraction (SBSE)}

Stir bar sorptive extraction (SBSE) was developed by Baltussen and co-workers in $1999{ }^{11}$. The SBSE device is made of a magnetic bar inserted into a glass tube coated with polydimethylsiloxane (PDMS). The technique can be performed by inserting the bar into a solution or its headspace; presently, there is a marketed version of SBSE, and it is GERSTEL® Multipurpose Sampler, because of which automation of the entire microextraction procedure is possible ${ }^{12}$. Following listed are some publications which shows a trend in usage of this technique for extraction of OPP from complex matrix.

Irma Lavagnini et al. developed Stir bar sorptive extraction (SBSE)-thermal desorption (TD) process accompanied with gas chromatography mass spectrometry (GC-MS) and the statistical variance component model (VCM) which was utilized for the determination of semi-volatile compounds involving organochlorine and organophosphorus pesticides in various synthetic and real fruit-based soft drink matrices. The sampling apparatus used was a stir bar (Twister, Gerstel, Muellheim, a/d Ruhr, Germany) $10 \mathrm{~mm}$ long, coated with $1.0 \mathrm{~mm}$ polydimethylsiloxane (PDMS) layer (63 L). In every sorption experiments the rotation speed was $1400 \mathrm{rpm}$ and the temperature kept $30 \circ \mathrm{C}$. The injection apparatus from Gerstel was made up using a multipurpose sampler (MPS), a thermal-desorption unit (TDU), a cooled injection system (CIS) and a programmed temperature vaporization injector (PTV). After which this unit was placed on an Agilent 6890 GC (Agilent Technologies, Little Falls, DE) connected to an Agilent 5975 quadrupole mass 
spectrometer. Analyses by GC was performed on a $30 \mathrm{~m} \times 0.25 \mathrm{~mm}$ i.d., $0.25 \mathrm{~m}$ film thickness VF-Xms column (Varian Inc., Lake Forest, CA). Helium was the carrier gas. Blank runs of the stir bar was carried out before and after each analysis to verify the absence of any carry-over effect. The technique showed an overall straight line during calibration for any analyte accounting for the uncertainty due to all the sources, namely, calibration runs performed at different times, matrixinduced deviations and measurement errors ${ }^{13}$.

Cong $\mathrm{Hu}$ et al. introduced a replacement technique incorporating stir bar sorptive extraction (SBSE) fiber with polydimethylsiloxane/polythiophene (PDMS/PTH) coating combined with liquid desorption-large volume injection-gas chromatography-flame photometric detection (LDLVI-GC-FPD) for the quantification of organophosphorus pesticides (OPPs, including phorate, fenitrothion, malathion, parathion and quinalphos) in water samples which may become environmental pollutant. Polythiophene was synthesized employing chemical oxidative polymerization method, and the PDMS/PTH coated stir bar was prepared by sol-gel technique. The preparation reproducibility of PDMS/PTH coated stir bar was observed to be good with the \%RSDs ranging from $3.9 \%$ to $8.1 \%(\mathrm{n}=7)$ in one batch, and from $5.9 \%$ to $14.9 \%(\mathrm{n}=6)$ among different batches. To improve execution of extraction, the working parameters affecting the extraction efficiency of SBSE, including desorption time, extraction time, stirring rate and ionic strength, were tested. Under the optimal conditions, the limits of detection $(\mathrm{S} / \mathrm{N}=3)$ was found to be in the range of $0.011-0.038 \mathrm{~g} / \mathrm{L}$ for the five target OPPs, and the linear range was from 0.2 to $100 \mathrm{~g} / \mathrm{L}$ for phorate and $0.1-100 \mathrm{~g} / \mathrm{L}$ for other four OPPs. The RSDs of the proposed method were in the range of $4.0-9.8 \%(\mathrm{n}=8, \mathrm{c}=1 \mathrm{~g} / \mathrm{L})$ and the enrichment factors were varied from 56.7 to 80.9-fold (theory enrichment factor was 100-fold). The proposed method was applied to the analysis of OPPs in East Lake water and ground water samples with recovery in the range of 77.7$119.8 \%$ and $79.1-109.8 \%$ for the spiked East Lake water and ground water samples, respectively 14.

\section{Dispersive Solid Phase Extraction}

Dispersive solid-phase extraction is one of the modifications of the SPE which consists of one of the steps of the QuEChERS method ("quick, easy, cheap, effective, rugged, and safe"). In this technique, an adsorbent-property is added to the solid in a liquid sample to extract contaminant; and particles are separated from the solution through centrifugation followed by desorption ${ }^{15}$. The credit for invention of this method rests with researcher Anastassiades et al., in the year 2003, initially employed for extraction and determination of pesticides in fruits and vegetables ${ }^{16}$. 
Miguel Ángel González-Curbelo et al have introduced a method for the estimation of about 15 organophosphorus pesticides, also including some of their metabolites, (disulfoton-sulfoxide, dimethoate, disulfoton, chlorpyrifos-methyl, malaoxon, terbufos, fenitrothion, pirimiphos-methyl, malathion, chlorpyrifos, disulfoton-sulfone and fensulfothion, terbufos-sulfone) in different types of commercial cereal based baby foods. Dispersive solid-phase extraction (dSPE) combined with multi-walled carbon nanotubes (MWCNTs) was incorporated along with gas chromatography with nitrogen phosphorus detection. Most suitable conditions involved a previously, ultrasound-assisted extraction of the sample with acetonitrile containing formic acid. After evaporating the extract and re-dissolving in water, a dSPE process was carried out with MWCNTs. The method was validated in terms of linearity, precision and accuracy, repeatability, and matrix effect. Absolute recoveries ranged from 64-105\% with \% RSD values below 7.6 \%. LOQ achieved ranged from 0.31 to 5.50 $\mu \mathrm{g} / \mathrm{kg}$, which were lower than the European Union maximum residue limits for pesticide residues in cereal-based baby foods ${ }^{17}$.

Guilong Peng et al have developed method based on dispersive solid phase extraction (DSPE) and dispersive liquid-liquid microextraction method based on solidification of floating organic droplets (DLLME-SFO) for the extraction of chlorpyrifos-methyl (CPM), chlorpyrifos (CP), and their main degradation product 3,5,6- trichloro-2-pyridinol (TCP) in tomato and cucumber samples. The determination was conducted by using high performance liquid chromatography with ultraviolet detection (HPLC-UV). In the DSPE-DLLME-SFO, the analytes were extracted using acetone. The clean-up of the extract carried out by DSPE by directly adding activated carbon sorbent into the extract solution, which was followed by shaking and filtration. Under the optimum conditions, the proposed method was sensitive and showed a good linearity within a range of 2$500 \mathrm{ng} / \mathrm{g}$, with the correlation coefficients (r) varying from 0.9991 to 0.9996 . The enrichment factors ranged from 127 to 138 . The limit of detections (LODs) were in the range of $0.12-0.68$ $\mathrm{ng} / \mathrm{g}$, and the relative standard deviations (RSDs) for $50 \mathrm{ng} / \mathrm{g}$ of each analytes in tomato samples were in the range of $3.25-6.26 \%(n=5)$. The proposed method was successfully applied for the extraction and determination of the mentioned analytes residues in tomato and cucumber samples, and satisfactory results were obtained ${ }^{18}$.

Xuejin Mao et al have developed a QuEChERS-type method without matrix interference to determine organophosphorus pesticide residues in edible vegetable oils, using UiO-66 as sorbent based on dispersive solid-phase extraction with cleanup. Microporous UiO-66 selectively adsorbed organophosphorus pesticides and excluded interfering compounds. Clean analytes were obtained by elution and analyzed using gas chromatography-tandem mass spectrometry. The 
dispersive solid-phase extraction conditions (amount of adsorbent, extraction time, desorption solvent volume, and elution time) were optimized. The LOD of the pesticides in vegetable oils were $0.16-1.56 \mathrm{ng} / \mathrm{g}$. Under optimized conditions, the average pesticide recoveries were 81.1-113.5\%. The intraday and interday relative standard deviations for analyte recovery ${ }^{19}$.

\section{Dispersive Solid Phase Microextraction (d- $\mu \mathrm{SPE})$}

The method of Dispersive solid phase microextraction is considered as a miniaturized and advanced version of dSPE. It was introduced to achieve high efficiency in microextraction using sorbents possessing high sorption capacity and large surface area ${ }^{15}$.

Qin Zhao et al have developed magnetic polypyrrole nanowires (mPPYs) and fabricated via a simple co-mixing method based on an "aggregation-wrap" mechanism. The polypyrrole (PPy) nanowires were synthesized by in situ chemical oxidative polymerization using cetyltrimethylammonium bromide as the "soft template" and the magnetic nanoparticles (MNPs) prepared using solvothermal methods. When these two nanomaterials were mixed in a solvent used vortex, the MNPs were wrapped into the PPy nanowire networks that formed during the aggregation process, leading to the formation of mPPYs which can be separated from the solvent quickly by a magnet. These mPPYs were applied for the extraction of 11 pesticides including organophosphorus, organochlorine, and pyrethroid using magnetic solid phase extraction (MSPE). Several parameters affecting the extraction efficiency were investigated, and under the optimized conditions, a simple and effective method for the determination of pesticide residues was established by coupling with gas chromatography/mass spectrometry (GC/MS) analysis. The whole pre-treatment process can be accomplished within $15 \mathrm{~min}$. The linearity range of the proposed method was $0.2-10 \mu \mathrm{g} / \mathrm{L}$, with correlation coefficients (R) of $0.995-0.999$; the limits of quantification for the target compounds were in the range of $0.09-0.29 \mu \mathrm{g} / \mathrm{L}$. In addition, an acceptable reproducibility was achieved by evaluating the intra- and inter-day precisions with relative standard deviations of less than 14 and $16 \%$, respectively. Finally, the established MSPEGC/MS method was successfully applied for the determination of pesticide residues in beverage teas, juices, and environmental water samples ${ }^{20}$.

Raheleh Hamedi et al have introduced amino-functionalized magnetic iron oxide nanoparticles coated with Sodium dodecyl sulfate as an sorbent particle for rapid preconcentration of three important organophosphorus pesticides, diazinon, chlorpyrifos, and phosalone, by ultrasoundassisted dispersive magnetic solid-phase microextraction. Fabrication of amino-functionalized magnetic nanoparticles was certified by characteristic analyses, including Fourier transform infrared spectroscopy, thermogravimetric analysis, scanning electron microscopy, and 
transmission electron microscopy. Affecting parameters on the removal efficiency were investigated and optimized through half fractional factorial design and Doehlert design, respectively. The analysis of analytes was performed by high-performance liquid chromatography with ultraviolet detection. Under the optimum conditions, extraction recoveries for $20 \mathrm{ng} / \mathrm{mL}$ of organophosphorus pesticides were ranged between of 84-97\% with preconcentration factors ranged from 134-155. Replicating the experiment in above condition for five times gave the relative standard deviations ${ }^{21}$.

Samaneh Akbarzade et al have introduced a technique of magnetic dispersive solid phase microextraction for extraction of organophosphorus pesticides (Fenitrothion, Malathion, Parathion and Diazinon) in water samples and juice samples with in line coupling of GC-MS. For sorbent, zero valent Fe-reduced graphene oxide quantum dots (rGOQDs@ Fe) was prepared and deployed for extraction of organophosphorus pesticides using MDSPME method. The workability of rGOQDs@ Fe was compared with magnetic graphene oxide nanocomposite and graphene oxide by recovery experiments of the organophosphorus pesticides. Parameters like donor phase volume, $\mathrm{pH}$ of donor phase, extraction time, stirring rate, and desorption conditions such as the type and volume of solvents and desorption time were thoroughly investigated and optimized. Under the optimal conditions, the technique showed a wide linear dynamic range with $\mathrm{R}$-square between 0.9959 and 0.9991 . The limit of detections, the intraday and inter day \%RSD $(n=5)$ were less than $0.07 \mathrm{ng} / \mathrm{mL}-1,4.7$, and $8.6 \%$, respectively. It was observed that the method was successfully employed for extraction and determination of organophosphorus pesticides in fruit juice samples (apple and grape juice) and real water samples (river, well and tap water). The obtained relative recoveries were in the range of $82.9 \%-113.2 \%$ with RSD percentages of less than $5.8 \%$ for all the real samples ${ }^{22}$.

\section{Microextraction With Packed Sorbent (MEPS)}

MEPS is a miniaturisation of SPE with being fully automated and coupled to in line chromatograph. The method was introduced by Mohamed Abdel-Rehim in the year $2004{ }^{23}$. The MEPS device contains a cartridge i.e. the extractive phase, within the body of a syringe. It requires sample volumes and solvents reduced to microliters.

C. Santos et al have developed a method for the determination of six organophosphorous pesticides (Ops) (azynphos-ethyl (AZP), diazinon (DZN), chlorpyrifos (CLP), chlorfenvinfos (CLF), parathionethyl (PRT) and quinalphos (QLP)) in whole blood incorporating microextraction by packed sorbent (MEPS) coupling along with gas chromatography-tandem mass spectrometry (GC-MS/MS). The optimization of the MEPS process was performed by designing of experiments 
(DOE) approach, evaluating various factors which mainly affected the extraction efficiency.

Ultimately, the number of sample strokes, wash volume, percentage of 2-propanol in the wash solvent and the number of elution strokes were successfully optimized using a response surface methodology (RSM). The developed and optimized method was fully validated according to international guidelines. Linearity was established from 2.5 to $50 \mu \mathrm{g} / \mathrm{mL}$ for AZP and from 0.5 to $50 \mu \mathrm{g} / \mathrm{mL}$ for the remaining compounds, with coefficients of determination (R2) higher than 0.99 in all cases. The lower limit of quantification was $2.5 \mu \mathrm{g} / \mathrm{mL}$ (AZP) and $0.5 \mu \mathrm{g} / \mathrm{mL}$ (remaining compounds). Recoveries ranged from $61 \%$ to $77 \%$. Intra and inter-day precision and accuracy were considered adequate according to the guidelines ${ }^{24}$.

\section{Fabric Phase Sorptive Extraction (FPSE)}

This is a newer technique which is an amalgamation of sol gel sorbent based microextraction combined with flexible fabric substrate. The microextraction device consisted of a synthetic or natural, flexible and permeable fabric substrate chemically coated with a sol-gel organic-inorganic hybrid sorbent in the form of ultra-thin coating for fast and sensitive separation ${ }^{25}$.

Ramandeep Kaur et al have developed a high-efficiency and solvent reduced microextraction technique, FPSE followed by gas chromatography and mass spectrometry analysis for the determination of four organophosphorus pesticides (malathion, terbufos, triazofos, and chlorpyrifos) in vegetable samples including tomato, brinjal, beans and cabbage. The study focussed on FPSE membrane, when immersed directly into the sample matrix, extracted target analytes even in conditions of high matrix interferences with simplification of sample preparation workflow. Most important FPSE parameters were investigated and optimized. The developed method showed acceptable linearity over the concentration range from $0.5-500 \mathrm{ng} / \mathrm{g}$. Under optimum conditions, the LOD was found range from 0.033 to $0.136 \mathrm{ng} / \mathrm{g}$. The $\% \mathrm{RSD}$ for the extraction of organophosphorus pesticides was obtained $<5 \%$. Subsequently, the new method was applied to beans, tomato, brinjal, and cabbage samples. The results from the real sample analysis indicate that the method is in alignment with green chemistry, rapid, and economically feasible for the determination of organophosphorus pesticides in vegetable samples ${ }^{26}$.

\section{CONCLUSION}

By considering the whole discussion on sample preparation miniaturized versions and modifications available so far for Organophosphorus pesticide residue extraction from complex matrix, its importance still remains vital in analytical procedure. As efficiency in this step ensures minimisation of error in the entire analytical process. From the review on various approaches discussed, it has been observed that dispersive miniaturisation e.g. dSPE and d- $\mu$ SPE, 
accompanied with automation micro extraction methods have been widely accepted for sample preconcentration due to their association with QuECHERS (Quick, easy, cheap, effective, rugged and safe) technique. Earlier, approaches showed use of stir bar sorptive variation of SPE with PDMS mostly used as sorbent for extraction of OPP. Newer techniques such as use of sol gel sorbents coated with fused cellulose substrate (an alternative to fused silica substrate) as the approach in FPSE has given a wider aspect to sample preparation for extraction of OPP, by solving many problems encountered like stripping or breakage of coating, swelling of fibers prevented in some organic solvents. Also, this miniaturisation resolves the need for more solvent, reduced sample volume, ensured less chances of error in analysis, thereby leading towards the green approach. The automation of entire sampling process also aided to minimization of error and coupling the process with in line gas chromatograph or liquid chromatograph assuring rapid, sensitive obtainment of result with comparatively reduced ambiguity.

Seeing the trend of developments in sample preparation employing miniaturisation in SPE for OPP extraction, it would be concluded that the focus would be more on modification pertaining to inventing more developed and efficient sorbents in order to achieve further considerable accuracy in extraction procedure.

\section{REFERENCES}

1. Medina DA, Cabal LF, Lanças FM, Santos-Neto ÁJ. Sample treatment platform for automated integration of microextraction techniques and liquid chromatography analysis. HardwareX. 2020 Feb 24:e00099.

2. Płotka-Wasylka J, Szczepańska N, de La Guardia M, Namieśnik J. Miniaturized solidphase extraction techniques. TrAC Trends in Analytical Chemistry. 2015 Nov 1;73:19-38.

3. Gałuszka A, Migaszewski Z, Namieśnik J. The 12 principles of green analytical chemistry and the SIGNIFICANCE mnemonic of green analytical practices. TrAC Trends in Analytical Chemistry. 2013 Oct 1;50:78-84.

4. Armenta S, Garrigues S, de la Guardia M. The role of green extraction techniques in Green Analytical Chemistry. TrAC Trends in Analytical Chemistry. 2015 Sep 1; 71:2-8.

5. Nazario CE, Fumes BH, da Silva MR, Lanças FM. New materials for sample preparation techniques in bioanalysis. Journal of Chromatography B. 2017 Feb 1; 1043:81-95.

6. Rutkowska M, Płotka-Wasylka J, Sajid M, Andruch V. Liquid-phase microextraction: A review of reviews. Microchemical Journal. 2019 Sep 1; 149:103989. 
7. Abdulra'uf LB, Chai MK, Tan GH. Applications of solid-phase microextraction for the analysis of pesticide residues in fruits and vegetables: A review. Journal of AOAC International. 2012 Sep 1; 95(5):1272-90.

8. Aulakh JS, Malik AK, Kaur V, Schmitt-Kopplin P. A Review on solid phase micro extraction-high performance liquid chromatography (SPME-HPLC) analysis of pesticides. Critical reviews in analytical chemistry. 2005 Jan 1; 35(1):71-85.

9. Arthur CL, Pawliszyn J. Solid phase microextraction with thermal desorption using fused silica optical fibers. Analytical chemistry. 1990 Oct 1; 62(19):2145-8.

10. Rahimi M, Bahar S, Heydari R, Amininasab SM. Determination of quercetin using a molecularly imprinted polymer as solid-phase microextraction sorbent and highperformance liquid chromatography. Microchemical Journal. 2019 Jul 1; 148:433-41.

11. Baltussen E, Sandra P, David F, Cramers C. Stir bar sorptive extraction (SBSE), a novel extraction technique for aqueous samples: theory and principles. Journal of Microcolumn Separations. 1999;11(10):737-47.

12. David F, Ochiai N, Sandra P. Two decades of stir bar sorptive extraction: A retrospective and future outlook. TrAC Trends in Analytical Chemistry. 2019 Mar 1; 112:102-11.

13. Lavagnini I, Urbani A, Magno F. Overall calibration procedure via a statistically based matrix-comprehensive approach in the stir bar sorptive extraction-thermal desorption-gas chromatography-mass spectrometry analysis of pesticide residues in fruit-based soft drinks. Talanta. 2011 Feb 15; 83(5):1754-62.

14. Hu C, He M, Chen B, Hu B. A sol-gel polydimethylsiloxane/polythiophene coated stir bar sorptive extraction combined with gas chromatography-flame photometric detection for the determination of organophosphorus pesticides in environmental water samples. Journal of Chromatography A. 2013 Feb 1; 1275:25-31.

15. Chisvert A, Cárdenas S, Lucena R. Dispersive micro-solid phase extraction. TrAC Trends in Analytical Chemistry. 2019 Mar 1; 112:226-33.

16. Anastassiades M, Lehotay SJ, Štajnbaher D, Schenck FJ. Fast and easy multiresidue method employing acetonitrile extraction/partitioning and "dispersive solid-phase extraction" for the determination of pesticide residues in produce. Journal of AOAC international. 2003 Mar 1; 86(2):412-31.

17. González-Curbelo MÁ, Asensio-Ramos M, Herrera-Herrera AV, Hernández-Borges J. Pesticide residue analysis in cereal-based baby foods using multi-walled carbon nanotubes 
dispersive solid-phase extraction. Analytical and bioanalytical chemistry. 2012 Jul $1 ; 404(1): 183-96$.

18. Peng G, He Q, Lu Y, Mmereki D, Zhong Z. Determination of organophosphorus pesticides and their major degradation product residues in food samples by HPLC-UV. Environmental Science and Pollution Research. 2016 Oct 1; 23(19):19409-16.

19. Mao X, Yan A, Wan Y, Luo D, Yang H. Dispersive Solid-Phase Extraction Using Microporous Sorbent UiO-66 Coupled to Gas Chromatography-Tandem Mass Spectrometry: A QuEChERS-Type Method for the Determination of Organophosphorus Pesticide Residues in Edible Vegetable Oils without Matrix Interference. Journal of agricultural and food chemistry. 2019 Jan 18;67(6):1760-70.

20. Zhao Q, Lu Q, Feng YQ. Dispersive microextraction based on magnetic polypyrrole nanowires for the fast determination of pesticide residues in beverage and environmental water samples. Analytical and bioanalytical chemistry. 2013 May 1; 405(14):4765-76.

21. Hamedi R, BG Aghaie A, Hadjmohammadi MR. Magnetic core micelles as a nanosorbent for the efficient removal and recovery of three organophosphorus pesticides from fruit juice and environmental water samples. Journal of Separation Science. 2018 May; 41(9):203745.

22. Akbarzade S, Chamsaz M, Rounaghi GH, Ghorbani M. Zero valent Fe-reduced graphene oxide quantum dots as a novel magnetic dispersive solid phase microextraction sorbent for extraction of organophosphorus pesticides in real water and fruit juice samples prior to analysis by gas chromatography-mass spectrometry. Analytical and bioanalytical chemistry. 2018 Jan 1; 410(2):429-39.

23. Abdel-Rehim M. New trend in sample preparation: on-line microextraction in packed syringe for liquid and gas chromatography applications: I. Determination of local anaesthetics in human plasma samples using gas chromatography-mass spectrometry. Journal of Chromatography B. 2004 Mar 5; 801(2):317-21.

24. Santos C, Oppolzer D, Gonçalves A, Barroso M, Gallardo E. Determination of organophosphorous pesticides in blood using microextraction in packed sorbent and gas chromatography-tandem mass spectrometry. Journal of analytical toxicology. 2018 Jun $1 ; 42(5): 321-9$.

25. Zilfidou E, Kabir A, Furton KG, Samanidou V. Fabric phase sorptive extraction: current state of the art and future perspectives. Separations. 2018 Sep; 5(3):40. 
26. Kaur R, Kaur R, Rani S, Malik AK, Kabir A, Furton KG. Application of fabric phase sorptive extraction with gas chromatography and mass spectrometry for the determination of organophosphorus pesticides in selected vegetable samples. Journal of separation science. 2019 Feb; 42(4)s:862-70.

AJPHR is

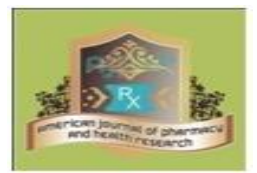

Peer-reviewed

monthly

Rapid publication

Submit your next manuscript at

editor@ajphr.com / editor.ajphr@gmail.com 